\title{
Dimensional transitions in small Yukawa clusters
}

\author{
A. Radzvilavičius, O. Rancova, and E. Anisimovas \\ Department of Theoretical Physics, Vilnius University, \\ Sauletekio al. 9-III, LT-10222 Vilnius, Lithuania
}

(Dated: April 21, 2022)

\begin{abstract}
We provide the detailed analysis of structural transitions leading to the rapid changes in dimensionality of small Yukawa clusters. These transformations are induced by the variations in the shape of confinement as well as the screening strength. We show, that even in the most primitive systems composed of only a few strongly interacting particles, the order parameter exhibits a power-law behavior in the vicinity of the critical point of the continuous transition. The critical exponent $\gamma=1 / 2$ is found to be universal in all studied cases, which is consistent with the general theory of continuous phase transitions.
\end{abstract}

PACS numbers: 61.46.Bc, 64.60.-i, 52.27.Lw

\section{INTRODUCTION}

The emergence of ordered patterns within complex systems of interacting entities draws attention of researchers in diverse fields, including physics, biology, mathematics and computer science [1]. Confined Wigner crystals are among the most primitive systems where the phenomenon of self-organization is observed. A small number of charged particles is placed into a confining potential well, where in the limit of low temperatures ordered structures are formed. This crystalline type of matter was successfully realized experimentally in a variety of well known systems, such as electrons on the surface of liquid helium [4, 5, cooled ions in traps 6] or strongly coupled particle clusters in complex plasmas 7 .

Systems of strongly coupled particles are of high scientific interest due to various collective phenomena, e.g. cooperative dynamics, waves and phase transitions. First order transitions between solid and liquid states of matter, namely melting and crystallization, were widely investigated in the studies of many-particle dusty plasma crystals, also known as Yukawa clusters [8, 9]. Crystalline structures formed by dust particles in complex plasmas turned out to be an extremely handy tool for these studies, as the convenient length and time scales, stability and transparency of these systems allow for direct optical observation and accurate measurements 7]. On the other hand, there is another type of transitions, occurring in the simplest few-particle systems confined by asymmetric traps. These are observed when a small change in one of the control parameters causes a sudden change in the dimensionality of the system, and therefore are called dimensional or zigzag phase transitions [10]. Note that these transitions take place in finite systems and are analogous to the second order phase transitions commonly defined and studied in the thermodynamic limit.

Dimensional transitions in small two-dimensional Yukawa clusters have been extensively studied in Ref. 11 both experimentally and numerically. The authors demonstrated an excellent agreement between the computed configurations of particles and the arrangements observed in complex plasma experiments. Structural zigzag transitions were induced by variations in the number of particles, the value of the Yukawa shielding parameter $\kappa$ or the shape of the confinement potential. A power-law behavior of the order parameter in the vicinity of phase transition was observed, and interpreted as a characteristic feature of second order phase transitions. However, the numerical values of critical exponents provided in 11 cast some doubt. These values are distinctly different from the classical mean-field value $1 / 2$ which one would naturally expect in a finite system of just a few particles. Therefore, one of the main motivations behind the current work is to provide the results of numerical modelling with higher precision and show the universality of critical exponents. We also extend the investigation to the clusters of different sizes and dimensional transitions of other types.

Our paper is organized as follows. In section II the model system is described and the procedure of our calculations is presented. Section III presents the results of the numerical modelling of dimensional transitions, grouped by their type in subsections. Main points of the article are summarized in section IV. Additionally, Appendix A provides details on the analytically solvable cases and exact values of critical parameters.

\section{NUMERICAL PROCEDURE}

We investigate numerically systems of $N$ identical particles of mass $m$ and charge $Q$, interacting through the Yukawa inter-particle potential. The interaction energy of two charges embedded in a screening environment thus reads

$$
V_{i j}=\frac{Q^{2}}{4 \pi \epsilon_{0}} \frac{\mathrm{e}^{-\kappa r_{i j}}}{r_{i j}},
$$

where $\kappa$ stands for the shielding strength (inverse screening length) and controls the range of interaction. Particles are kept together by a harmonic confinement potential $V_{\mathrm{c}}(\mathbf{r})=\frac{1}{2} m \omega_{0}^{2}\left(x^{2}+\alpha^{2} y^{2}+z^{2}\right)$ in $3 \mathrm{D}$ or $V_{\mathrm{c}}(\mathbf{r})=$ $\frac{1}{2} m \omega_{0}^{2}\left(x^{2}+\alpha^{2} y^{2}\right)$ in $2 \mathrm{D}$. The anisotropy parameter $\alpha$ controls the shape of the confinement, which reduces to 
the symmetric spherical or circular form at $\alpha=1$, and takes the shape of oblate $(\alpha>1)$ or prolate $(\alpha<1)$ spheroid in 3D and an ellipse in 2D.

In the regime of strong correlations the potential energy dominates over the kinetic one, and the total energy of the model system is given by

$$
U\left(\mathbf{r}_{1}, \ldots, \mathbf{r}_{N}\right)=\sum_{i=1}^{N} \frac{1}{2}\left(x_{i}^{2}+\alpha^{2} y_{i}^{2}+z_{i}^{2}\right)+\sum_{i>j}^{N} \frac{1}{r_{i j}} \mathrm{e}^{-\kappa r_{i j}} .
$$

The units of length and energy are conveniently chosen as $r_{0}=\left(Q^{2} / 4 \pi \epsilon_{0} m \omega_{0}^{2}\right)^{1 / 3}$ and $E_{0}=Q^{2} / 4 \pi \epsilon_{0} r_{0}$. Obviously, $\kappa$ is now measured in $r_{0}^{-1}$. In two dimensions, the $z$ coordinates of all particles are set to zero, so that all the particles lie within the $(x y)$ plane.

Stable arrangements of particles correspond to the local and global minima of the potential energy (2). In our present work, stationary states are located by the method of multiple heating-relaxation cycles, based on the Monte Carlo and numerical minimization algorithms. The method was already proven to be efficient and reliable in our previous studies 12. As it turned out, the potential energy landscape of $(2)$ is rather complex even for small values of $N$ and might be described as a collection of local minima, separated by potential barriers of various heights. In a first stage of the algorithm, thermalization takes place, that is, the system is heated to the temperature high enough to overcome all the potential barriers. This stage is accomplished by performing a large number (few thousand) of Metropolis Monte Carlo steps [13], which leads to a configuration, that may be regarded as drawn randomly from the Boltzmann distribution corresponding to the given temperature. Each minimum controls a certain area of coordinate space, called basin of attraction. The area of attraction varies from basin to basin, which means that different stable states are realized with different probabilities [12, 14. Some of the minima are located within the regions with steep walls, while others lie in broad shallow valleys and therefore require a considerable effort to find. In a second stage of the computational procedure, the temperature of the system is suddenly set to zero and the closest local minimum of potential energy is located by employing the steepest descent and Newton optimization techniques. As frequently there is more than one local minimum [12, the whole cycle of thermalization and relaxation is repeated a large number of times, to ensure that all of the basins are visited and all stationary points of $(2)$ are revealed.

Departure of the anisotropy parameter $\alpha$ from unity breaks spherical (or circular in 2D) symmetry and, as a result, prolate or flattened structures are formed. Eventually, at the critical value of parameter $\alpha_{\mathrm{c}}$, dimensional transitions are observed, three-dimensional clusters become planar, while two-dimensional structures are transformed into linear ones. In order to determine the critical values of $\alpha$ with high precision, we repeat our calculations by incrementing $\alpha$ in small steps. As it will become evident shortly, properties of Yukawa clusters, including critical values of the anisotropy parameter and critical exponents, depend strongly on the screening parameter $\kappa$. Therefore, in most cases we use four different values of Yukawa screening strength, $\kappa=0,1,2,3$. In the simplest case of $\kappa=0$, inter-particle potential reduces to the simple unscreened Coulomb interaction.

A second order phase transition is marked by a sudden appearance or disappearance of some property of the system, called an order parameter, in response to a small change in a control parameter. We investigate dimensional phase transitions by keeping an eye on the total potential energy and order parameter $\langle y\rangle$, which is defined as the root mean square of the coordinate $y$ :

$$
\langle y\rangle=\left(\frac{1}{N} \sum_{i=1}^{N} y_{i}^{2}\right)^{1 / 2}
$$

Naturally, dimensional transitions are signified by a sudden change of $\langle y\rangle$ to zero. In particular, $\langle y\rangle$ is a good choice for an order parameter, since $\langle y\rangle=0$ in the $1 \mathrm{D}$ (2D) configuration and $\langle y\rangle>0$ in the 2D (3D) configuration. The state variables that determine the system configuration are then $N, \kappa$ and $\alpha$.

\section{RESULTS}

\section{A. $2 \mathrm{D} \rightarrow 1 \mathrm{D}$ transitions}

We first investigate the simplest few-particle twodimensional systems, undergoing $2 \mathrm{D} \rightarrow 1 \mathrm{D}$ structural transitions. In the case of $\alpha=1$, the confinement potential is symmetric and particles form ordered states, that were previously observed experimentally and modelled theoretically [15, 16. Systems with $N=3,4,7$ particles form only one stable configuration (ground state), while clusters with $N=5,6$ particles in symmetric traps support both ground- and one metastable configurations. Various states can be represented by listing the occupation numbers of different shells - ground state of 5particle system is therefore the configuration $(0,5)$ and metastable state is $(1,4)$ (for the arrangements of particles see Figure 1). As the anisotropy parameter $\alpha$ departs from unity, metastable states can become ground states, some states can disappear and new ones appear. In all investigated cases, however, there is only one stationary configuration near the dimensional transition - a zigzag shaped pattern, which soon becomes a 1D linear chain of particles at $\alpha>\alpha_{\mathrm{c}}$.

In the simplest symmetric case of $N=3$, particles form an equilateral triangle in the $(x y)$ plane. As the value of $\alpha$ increases, the triangular configuration is gradually deformed until the transition occurs at $\alpha=\alpha_{\mathrm{c}} \approx 1.55$, as shown in Figure 1 with $\kappa=0$. In fact, dimensional transition in three-particle system can be modelled analytically, which gives the value of $\alpha_{\mathrm{c}}=\sqrt{12 / 5}$ (see Appendix A. . Numerical simulation gives exactly the same value. 


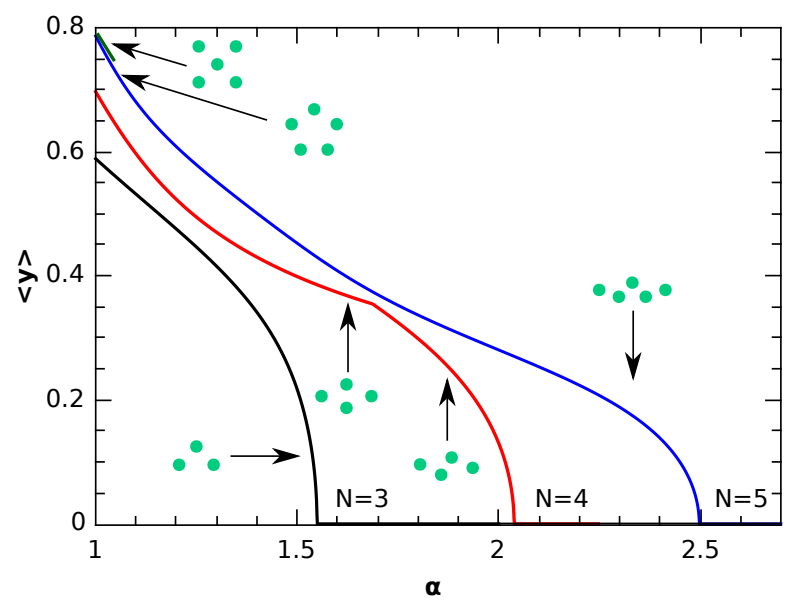

Figure 1. Value of the order parameter $\langle y\rangle$ as a function of the anisotropy $\alpha$ for $2 \mathrm{D}$ clusters with $N=3-5$ particles. Insets show typical arrangements of particles in various stages of compression. Note how quickly metastable state $(1,4)$ disappears (top left corner).

The structural transition is slightly more intriguing in the case of $N=4$ particles. As it can be deduced from the evolution of $\langle y\rangle$ in Figure 1, there is a discontinuity in a first derivative of the order parameter $\mathrm{d}\langle y\rangle / \mathrm{d} \alpha$ at the value of anisotropy parameter $\alpha \approx 1.69$. As it turns out, there are two stages of the four-particle cluster compression. In the first, slow stage, four particles form a rhombus-shaped structure, with the particles located exactly on $x$ or $y$ axis. Later, the stage of rapid compression takes over, with two particles departing from the line $x=0$ and forming a zigzag shaped pattern. Two typical rhombus- and zigzag-shaped configurations are presented in the insets of Figure 1. As it was shown in the previous study, the transition between these two stages is followed by the specific oscillation of the heat capacity [17. Analogous scenario applies to the other clusters with even number of particles. The dimensional transition is observed at $\alpha_{\mathrm{c}} \approx 2.04$, where $\langle y\rangle$ suddenly drops to zero.

Two competing configurations are first observed in the case of $N=5$ particles, namely states $(0,5)$ and $(1,4)$. As it is depicted in Figure 1. metastable state $(1,4)$ exists only in the narrow window of anisotropy, $1<\alpha<1.05$. On the other hand, the pentagonal ground state undergoes a continuous structural transformation, forms a zigzag-shaped cluster and finally becomes linear at $\alpha_{\mathrm{c}} \approx 2.50$.

Structural transitions become more complex for the systems with $N \geq 6$. Six particles in a symmetric confinement can form two stable states. As $\alpha$ increases, metastable $(0,6)$ state vanishes at $\alpha=1.05$ only to reappear again and become a new ground state later. The former ground state $(1,5)$ then disappears completely near $\alpha=1.22$. Six- and eight-particle clusters both feature the same discontinuity in $\mathrm{d}\langle y\rangle / \mathrm{d} \alpha$ as four-particle system, discussed above.

We have already seen in Figure 1, that critical value of the parameter $\alpha_{\text {c }}$ increases with $N$, when inter-particle interaction is of the Coulomb type. As it might be expected, $\alpha_{c}$ also grows as Yukawa potential screening parameter $\kappa$ is increased, which is shown in Figure 2 for $N=3-6$. The critical value of the anisotropy increases rapidly for $\kappa<1.5$ and almost saturates for high values of screening, i.e. $\kappa>4.0$.

The lines in Figure 2 represent boundaries between different phases of clusters. The structures are twodimensional below the line and form linear configurations above. Although we devote most of the present work to the transitions induced by deformations of the confinement well, structural changes can actually be caused by variations in any of three parameters $\alpha, \kappa$ or $N$. As the figure shows, two-dimensional cluster can become linear without any changes in $\alpha$, for example, when a value of $\kappa$ is diminished, or when a particle is removed from the system.

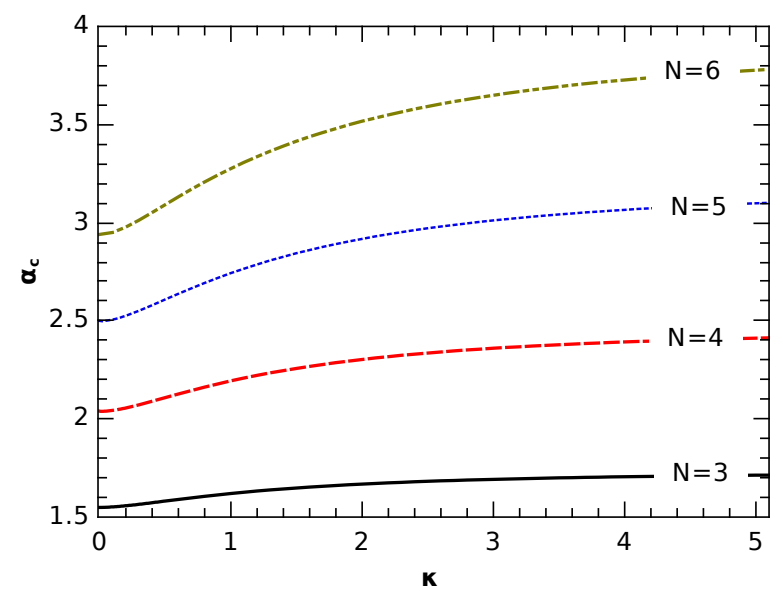

Figure 2. Phase diagram for two-dimensional Yukawa clusters of $N$ particles. Below the corresponding line, the cluster of $N$ particles is two-dimensional and one-dimensional above. We see, that dimensional transitions can be induced by variations in any of three parameters $\alpha, \kappa$ or $N$.

We further examine the power-law behavior of the order parameter $\langle y\rangle$ near its critical point $\alpha_{\mathrm{c}}$ in more detail. The power law is easily identified by plotting the logarithm of the order parameter, $\lg (\langle y\rangle)$, as a function of $\lg \left(\alpha_{\mathrm{c}}-\alpha\right)$. The function turns out to be linear for small values of $\left(\alpha_{\mathrm{c}}-\alpha\right)$. This observation confirms that in the vicinity of the transition point, the order parameter $\langle y\rangle$ demonstrates a power-law behavior, which is a typical property of second-order phase transitions:

$$
\langle y\rangle \propto\left(\alpha_{\mathrm{c}}-\alpha\right)^{\gamma}
$$

We determine the values of the exponent $\gamma$ near the critical point by analyzing the slope of the above discussed log-log plot. Namely, we take the numerical 
derivative of the function $\lg (\langle y\rangle)=f\left(\lg \left(\alpha_{\mathrm{c}}-\alpha\right)\right)$. Calculated exactly at the critical point this derivative yields the exact 'theoretical' value of the critical exponent. However, in an experimental or numerical investigation the precise location of the critical point may not be known. Thus, calculating the numerical derivative a bit away from the critical point we are able to mimic the uncertainty and errors present in a realistic experimental situation. It turns out, that in all cases, $\gamma=1 / 2$ as long as $\alpha$ is close to its critical value $\alpha_{\mathrm{c}}$ (Figure 3 . However, the local value of the exponent (determined as the numerical derivative) is very sensitive to the deviation of anisotropy parameter from $\alpha_{\mathrm{c}}$.

Figure 3 shows the dependence of the power-law exponent $\gamma$ on the deviation of $\alpha$ from its critical value. The case of $2 \mathrm{D}$ cluster with $N=3$ particles and four different values of screening length is presented. We see, that $\gamma$ departs from the value of $1 / 2$ significantly when the deviation from $\alpha_{\mathrm{c}}$ reaches third decimal and attains its minimum near the first decimal. Furthermore, the exponent $\gamma$ attains significantly lower values far from $\alpha_{\mathrm{c}}$ in the systems with stronger screening. Other than that, there are no qualitative differences in the critical behavior of systems with different values of $\kappa$. The exponent $\gamma$ of the other systems with $N>3$ behaves similarly to the case of three particles presented here.

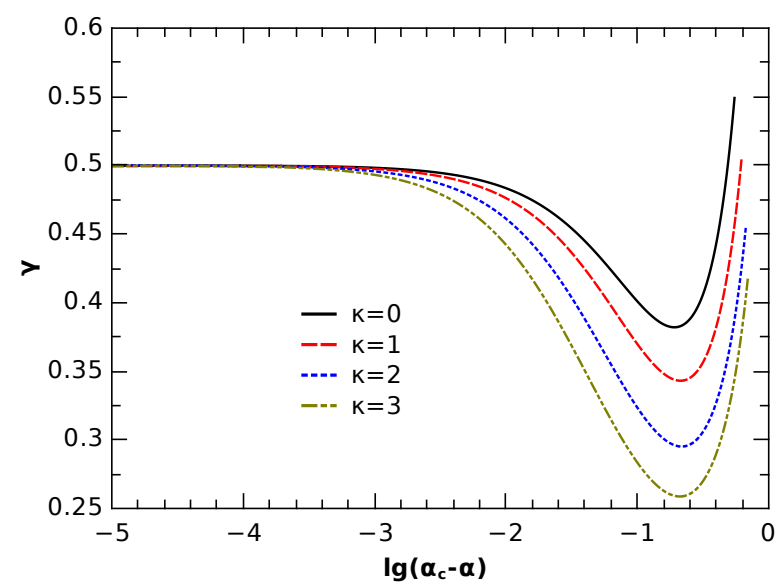

Figure 3. Critical exponent of the transition observed in 2D three-particle systems as a function of deviation of $\alpha$ from its critical value $\alpha_{\mathrm{c}}$ for different strengths of screening.

A thorough analysis of transitions in a planar cluster of five particles was reported by Sheridan in 11. The given value of critical parameter is $\alpha_{\mathrm{c}}=2.96$ and the critical exponent is said to be $\gamma=0.39 \neq 1 / 2$, while we find critical anisotropy parameter to be $\alpha_{\mathrm{c}}=3.01$. According to the results of our calculations, a value of $\alpha=2.96$ corresponds to the exponent $\gamma=0.37$, which is close to the value reported by Sheridan et. al. Therefore, the reason of differences between the results, published in [11] and our discoveries almost undeniably lies in the extreme sensitivity of $\langle y\rangle$ on the value of anisotropy parameter and distance from its critical value. Thus, the accuracy of the results presented in [11] is probably not sufficient.

As it was demonstrated in 11 and shown in Figure 2. continuous transitions might also be induced by the variations in the screening strength $\kappa$. By keeping $N$ and $\alpha$ constant, we gradually change a value of $\kappa$ while tracking the changes parameter $\langle y\rangle$ undergoes. The dimensional transition takes place when the value of $\langle y\rangle$ suddenly drops to zero, at which point the critical value of $\kappa$ is obtained (see the inset of Figure 4). It turns out, that $\langle y\rangle$ exhibits the same power law behavior near the transition, i.e. $\langle y\rangle \propto\left(\kappa-\kappa_{c}\right)^{\beta}$. Figure 4 shows the dependence of critical exponent $\beta$ on a logarithm of the distance from the critical value $\kappa_{c}$ for three systems. As opposed to the results presented in [11, we see again, that in all cases close to the transition point $\beta=1 / 2$. Moving away from the critical point, however, exponent $\beta$ departs from the value of $1 / 2$ significantly.

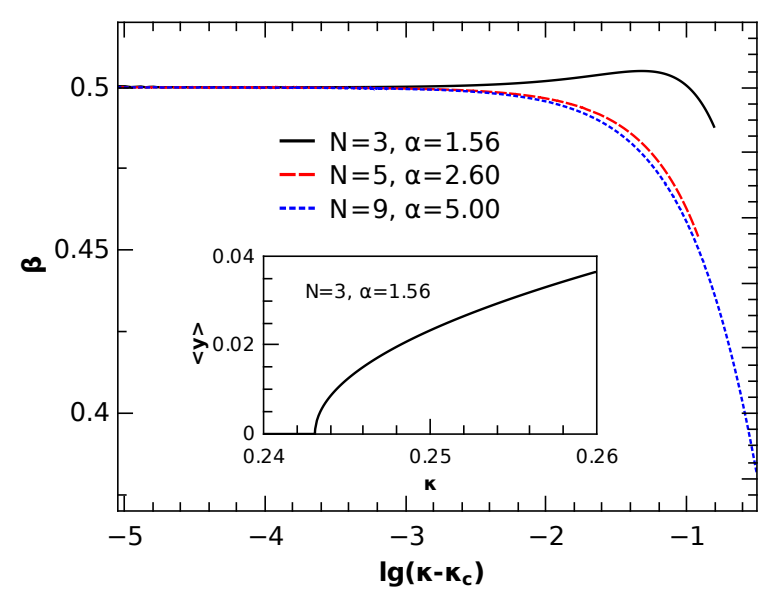

Figure 4. Critical exponent $\beta$, as a function of deviation of $\kappa$ from its critical value $\kappa_{\mathrm{c}}$ for three $2 \mathrm{D}$ clusters. The evolution of the order parameter $\langle y\rangle$ close to the dimensional transition (inset).

\section{B. $3 \mathrm{D} \rightarrow 2 \mathrm{D}$ transitions}

We further investigate structural transitions in threedimensional Yukawa clusters with $N=4$ to $N=8$ particles and integer values of screening parameter up to $\kappa=3$. Increased values of the parameter $\alpha$, turn initially spherical structure into the oblate one; eventually, after the anisotropy parameter reaches its critical value $\alpha_{\mathrm{c}}$, dimensional phase transition takes place and familiar twodimensional clusters are formed. In three-dimensional transformations of five- and six-particle clusters, two different final states are possible, as opposed to the zigzag transitions in $2 \mathrm{D}$, where only one linear configuration can be formed. Therefore, in $3 \mathrm{D} \rightarrow 2 \mathrm{D}$ transitions, there is 
a distinct value of $\alpha_{\mathrm{c}}$ for each final configuration and we are concerned by the properties of phase transitions of a particular stable state.

As the confinement potential well is squeezed in $y$ direction, it is handy to label small clusters according to the arrangement of particles in the projection to $(x z)$ plane, in a manner similar to the state labeling by shell occupation numbers in two dimensions. Moreover, particles in three-dimensional anisotropic traps frequently organize themselves within the layers, parallel to the $(x z)$ plane. For the sake of clarity and unambiguous definition of the configurations, we will also use a list of particle numbers in distinct layers, enclosed within curly brackets.

The simplest system, undergoing a non-trivial $3 \mathrm{D} \rightarrow$ $2 \mathrm{D}$ transition is the cluster composed of four particles. Not surprisingly, four particles in a symmetric threedimensional trap form a regular tetrahedron, and there is only one possible square-shaped $(0,4)$ state in two dimensions. Figure 5 shows dependence of the order parameter $\langle y\rangle$ and potential energy of a system $E$ on the anisotropy parameter $\alpha$. We see, that $\langle y\rangle$ changes continuously and the transition is remarkably similar to the one in $2 \mathrm{D}$ case of $N=3$ particles. The potential energy gradually increases as the potential trap is flattened, until a two-dimensional structure is formed at $\alpha_{\mathrm{c}} \approx 1.22$. As it is demonstrated in Appendix A, this symmetric transition can be modelled analytically; the critical value turns out to be $\alpha_{\mathrm{c}}=(4 \sqrt{2} /(1+2 \sqrt{2}))^{1 / 2}$ — exactly the same as determined in our numerical modelling. Naturally, the value $\alpha_{\mathrm{c}}$ is sensitive to the range of the interparticle Yukawa potential. As Figure 8 shows, critical value of the anisotropy parameter increases rapidly with the strength of screening for $\kappa<2$ and significantly slower after that, thus reminding of the transitions from two- to one-dimensional configurations.

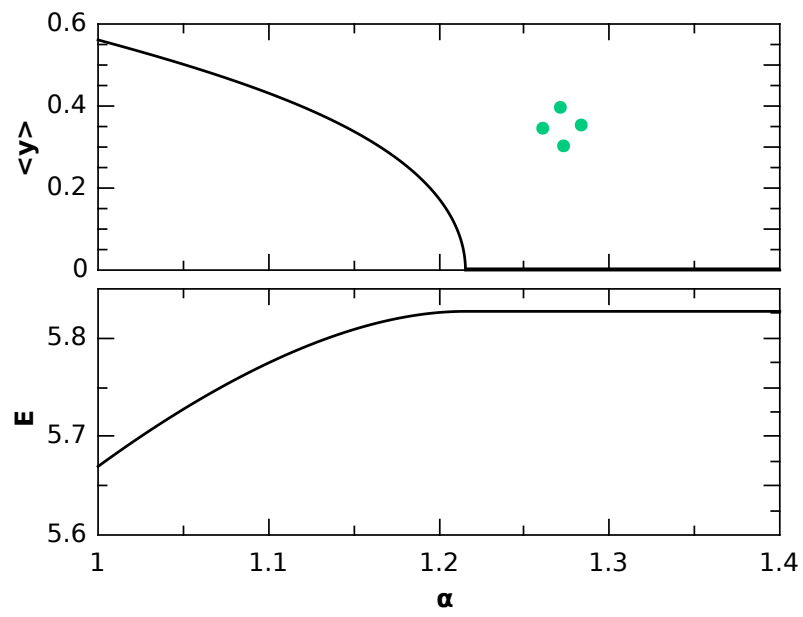

Figure 5. Order parameter $\langle y\rangle$ and potential energy $E$ of a four-particle 3D Coulomb cluster in the asymmetric potential trap with anisotropy parameter $\alpha$.

As it was already pointed out before, there are two competing stable states observed in a two-dimensional system with $N=5$ particles. Five particles in a spherically symmetric 3D confinement potential, however, can form only one stable configuration. Slightly increased anisotropy leads to the formation of a three-layer structure, with the arrangement of particles within these layers being $\{2,2,1\}$. As parameter $\alpha$ increases above the value of $\alpha=1.05$, two layers merge forming a square and thus transforming the configuration into a pyramidal structure $\{4,1\}$ with projection $(1,4)_{x z}$. This structural transition from three-layered cluster to the pyramidal configuration is signified by the discontinuity in the derivative $\mathrm{d}\langle y\rangle / \mathrm{d} \alpha$ (Figure 6).

As it is demonstrated in Figure 6 for a pure Coulomb interaction, a second stable state appears when the anisotropy parameter reaches the value of $\alpha_{0} \approx 1.29$. A new pentagonal state undergoes an asymmetric dimensional transition and is soon transformed into the new ground state $(0,5)$. The metastable configuration $(1,4)_{x z}$, on the other hand, becomes two-dimensional only at $\alpha_{\mathrm{c}} \approx 1.60$, through the so-called "pyramidal" transition mechanism.

Both point of appearance of the second state in fiveparticle system $\alpha_{0}$, and its critical value $\alpha_{\mathrm{c}}$ depends on the type of the interaction potential and its screening parameter $\kappa$. As it is shown in Figure 8, both parameters grow with the strength of screening. The distance between $\alpha_{0}$ and $\alpha_{\mathrm{c}}$, however, rapidly diminishes. As the screening reaches the value of $\kappa \approx 4.5$, two lines merge and a new stable state appears already in its twodimensional pentagonal form $(0,5)$.

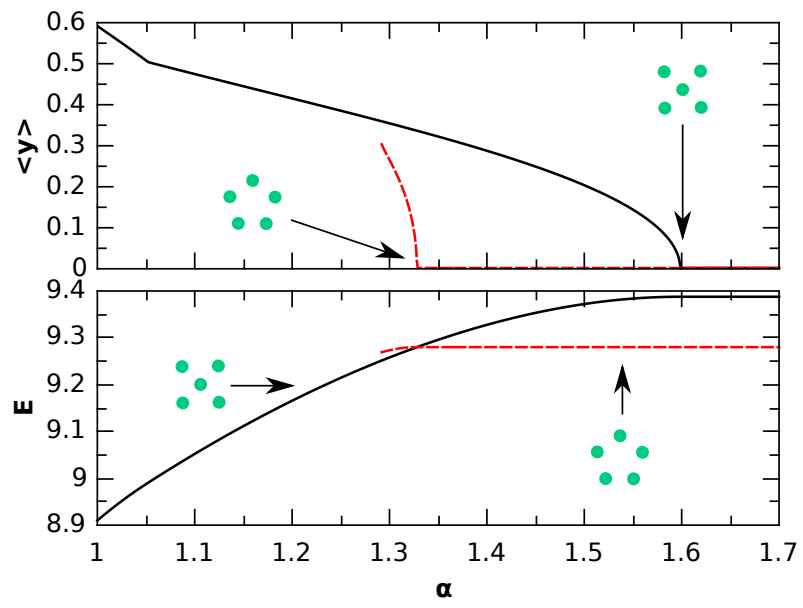

Figure 6. Order parameter $\langle y\rangle$ and potential energy $E$ of a 5particle 3D Coulomb cluster in the asymmetric potential trap with anisotropy parameter $\alpha$. Here and later the insets show projections of configurations to $(x z)$ plane.

A pyramidal configuration might be described as a planar base, composed of $n=4-6$ particles lying parallel to the $(x z)$ plane, and a single particle located right above the center of the base, that is, configuration $\{N-1,1\}$, 
$(1, N-1)_{x z}$. A pyramidal structural transition takes place, when the apex of the polyhedron is pushed into the base, thus becoming a two-dimensional configuration with only one particle in the center. A typical behavior of the order parameter $\langle y\rangle$ during such transitions was already discussed and is presented in figures 6 and 7. As a matter of fact, the dimensional transitions of a pyramidal type can be modelled analytically and exact values of critical parameters $\alpha_{\mathrm{c}}$ can be found, see Appendix A.

Even more stable configurations are observed in clusters with $N=6$ particles, as Figure 7 shows for the Coulomb inter-particle potential. Evolution of the system starts with a single stable state in the symmetric 3D trap - the octahedral configuration (full line in Figure 7). As the parameter $\alpha$ increases, this bipyramid is deformed by pushing two of its particles lying exactly on $y$-axis, towards each other, thus lowering the height and forming a configuration $\{1,4,1\}$ with projection $(1,4)_{x z}$. $\langle y\rangle$ decreases slowly, until the said two particles start to depart form the $y$-axis near $\alpha \approx 1.46$, at which point a phase of rapid deformation begins. Unfortunately, right after this happens, the stable state disappears. The same scenario of bipyramidal deformation also applies to the larger clusters, e.g. $N=7,8,9$, and has a specific, well recognizable shape of its $\langle y\rangle=f(\alpha)$ curve, with the segments of slow and rapid changes.

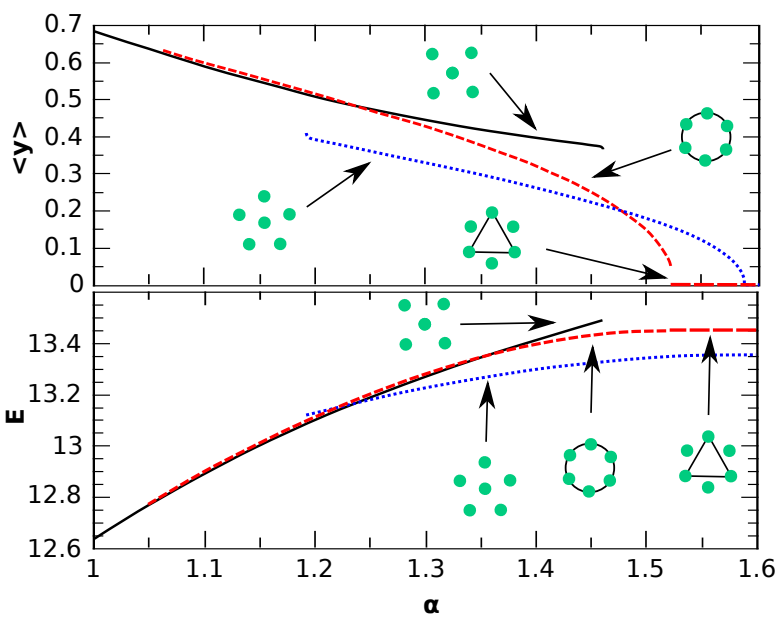

Figure 7. Order parameter $\langle y\rangle$ and potential energy $E$ of the six-particle 3D Coulomb cluster in the asymmetric potential trap with anisotropy parameter $\alpha$.

A new metastable state emerges near $\alpha \approx 1.06$ : the particles lie on the six vertices of two parallel equilateral triangles, centered precisely on $y$-axis and rotated by $\pi / 3$ with respect to each other, that is, state $\{3,3\}$ and $(0,6)_{x z}$. These triangular layers are pushed towards each other by deformations of the confinement, however, they fail to ever become a truly two-dimensional configuration. Instead, as it is demonstrated in Figure 7. the configuration ceases to exist at $\alpha \approx 1.52$ where the r.m.s. value of $y$ coordinate is still $\langle y\rangle \approx 0.05>0$. However, right before the disappearance, a new similar purely two-dimensional state shows up. The new planar configuration is composed of six particles lying on the vertices of two triangles of slightly different sizes (see Figure 7). Therefore, in a brief range of $\alpha$ values these two states exist simultaneously and there is no continuous transition between them. Finally, a pyramidal configuration $\{5,1\}$ appears near $\alpha \approx 1.19$ and undergoes the usual pyramidal dimensional transition at $\alpha_{\mathrm{c}} \approx 1.59$, the value predicted by our analytical model (appendix A).

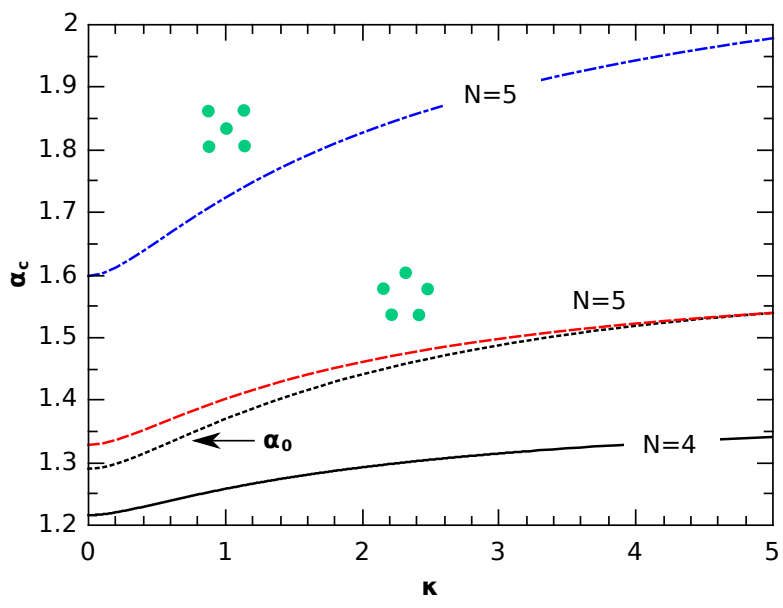

Figure 8. Critical value of anisotropy parameter $\alpha_{c}$ as a function of $\kappa$ in three-dimensional configuration with $N=4$ particles and two states of $N=5$ clusters. The line marked as $\alpha_{0}$ corresponds to the appearance of the metastable state in a five-particle cluster.

Close to the critical point of continuous transitions from three- to two-dimensional systems, a power-law behavior of the order parameter is detected once again, i.e. $\langle y\rangle \propto\left(\alpha_{\mathrm{c}}-\alpha\right)^{\gamma}$. In the same manner as in 2D case, Figure 9 shows the dependence of the power-law exponent $\gamma$ on the logarithm of $\alpha_{\mathrm{c}}-\alpha$. It turns out, that in a close vicinity of transition point, the critical exponent $\gamma=1 / 2$ does not depend on the screening strength $\kappa$. Deviations from this value occur when the departure of $\alpha$ from its critical value reaches third decimal. Just as in the two-dimensional case, the value of a critical exponent is lower for systems with stronger inter-particle potential screening, and drops as low as $\gamma \approx 0.35$ for $\kappa=3$. Essentially the same behavior of the exponent $\gamma$ is observed in larger three-dimensional systems, where dimensional transitions take place, be it pyramidal transitions or transformations of any other type.

As the number of particles grows, more and more stable states emerge and, as a consequence, $\langle y\rangle=f(\alpha)$ graphs become convoluted and somewhat difficult to study. An illustrative example is given in the inset of Figure 10 . where the behavior of the order parameter in stable states of 20-particle Coulomb system is presented. We can still see a few distinct continuous phase transitions in the vicinity of $\alpha \approx 2.26$, however different lines become hardly distinguishable at the lower values of $\alpha$. In very 


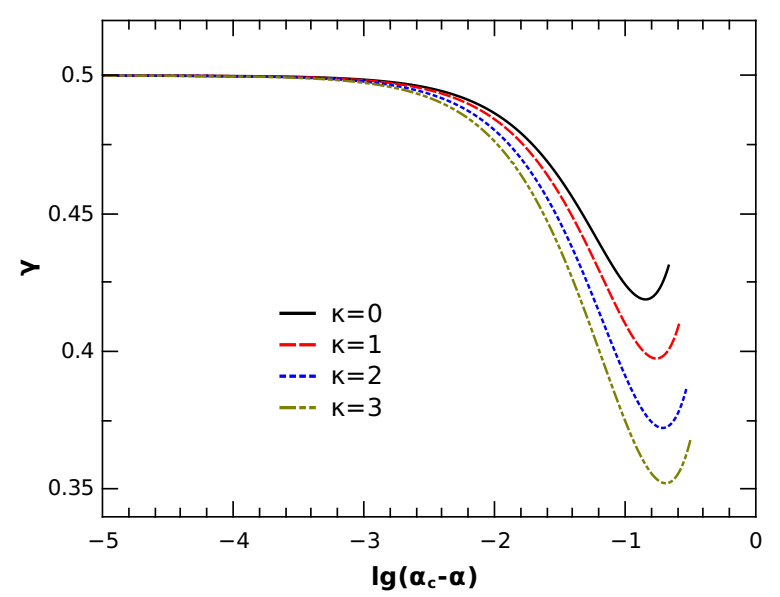

Figure 9. Critical exponent $\gamma$ for a dimensional transition, observed in 3D four-particle systems as a function of deviation of $\alpha$ from its critical value $\alpha_{\mathrm{c}}$ for different strengths of screening.

large systems, the values of $\langle y\rangle$ for all metastable states lie virtually on the same line, as it is illustrated in Figure 10 with a 100-particle cluster and $\kappa=0$.

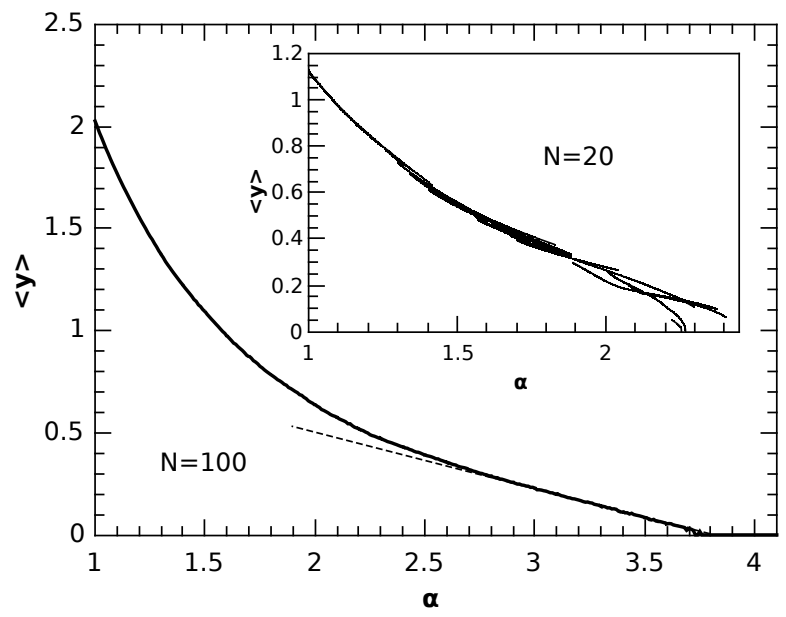

Figure 10. Order parameter $\langle y\rangle$ of large Coulomb systems with $N=100$ and $N=20$ (inset) particles as a function of the anisotropy $\alpha$.

It might be worth discussing the structural evolution of Yukawa clusters confined by traps with a prolate equipotential surface. In our model, this effect is achieved by lowering the value of anisotropy parameter $\alpha$ towards zero. In that way, elongated clusters are formed, with low potential energies and high values of $\langle y\rangle$. Consequently, in order to study this type of structural transitions, a new order parameter must be defined. We choose to rely on the root mean square of the distance from $y$-axis:

$$
\langle\rho\rangle=\left(\frac{1}{N} \sum_{i=1}^{N}\left(x_{i}^{2}+z_{i}^{2}\right)\right)^{1 / 2} .
$$

As Figure 11 shows for $N=3-6$, dependencies of the order parameter $\langle\rho\rangle$ on the anisotropy $\alpha$ are not smooth and in some cases feature discontinuities. We conclude, that in fact there is no direct transition from three- to one-dimensional configurations. Instead, the system is first transformed into the elongated 2D zigzag pattern, and only later, 2D $\rightarrow 1 \mathrm{D}$ structural transition takes place. With that in mind, there is no surprise, that the values of critical parameters $\alpha_{\mathrm{c}}$ found by lowering $\alpha$ are the exact inverses of those, determined in subsection IIIA.

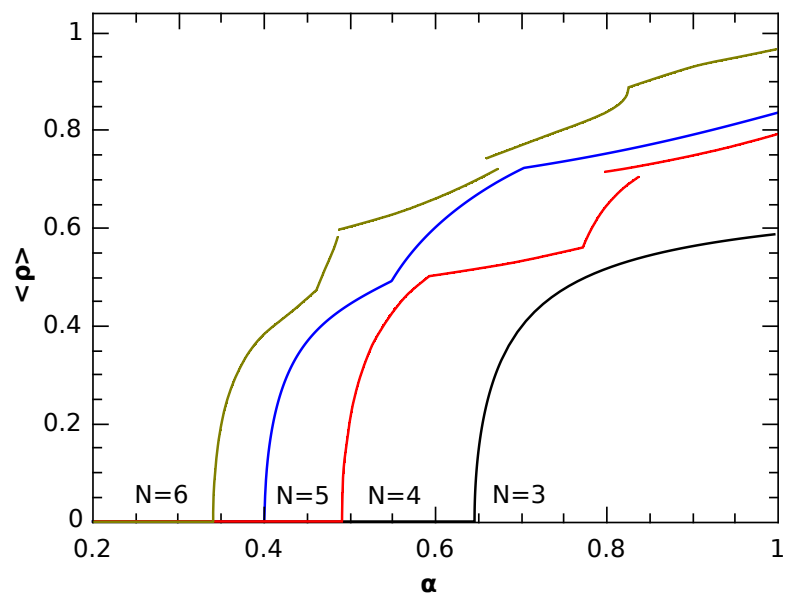

Figure 11. Order parameter $\langle\rho\rangle$ as a function of the anisotropy $\alpha<1$ for Coulomb clusters with $N=3-6$ particles.

Large three-dimensional clusters in prolate traps become one-dimensional through the mechanism, which seems to be universal for all values of $N$ used in our modelling. At first, the system is squeezed and elongated until the particles arrange themselves into the shape of double-helix. As $\alpha$ is lowered further, a number of helical turns decreases, until the helix unwinds and cluster becomes a two-dimensional zigzag configuration. 2D system then undergoes the usual zigzag transition with the power-law behavior near the critical point.

\section{CONCLUSION}

Confined Yukawa clusters are among the physical systems, where simple interparticle interactions lead to the emergence of complicated patterns and spontaneous ordering. In this article, we present our findings in the numerical and analytical studies of two- and threedimensional clusters confined by asymmetric parabolic traps. 
We confirm, that dimensional transitions from oblate three- to two-dimensional systems as well as from planar to linear configurations can be induced by changes in the anisotropy of the confinement $\alpha$, and screening strength $\kappa$. On the other hand, there are no direct transitions from three- to one-dimensional systems in prolate harmonic traps; two-stage transformations take place instead.

A critical value of the anisotropy parameter in general grows with the screening strength $\kappa$. The growth is steepest for small values of $\kappa$ and almost saturates for the large ones. In a close vicinity of dimensional phase transition, the order parameter $\langle y\rangle$ exhibits power-law dependence on a control parameter, be it $\alpha$ or $\kappa$.

In all cases studied here, the critical exponent is found to be universal and equal to $1 / 2$, which is consistent with the general theory of second order phase transitions. However, a value of the power-law exponent turns out to be very sensitive to the deviations of a control parameter from its critical value. Far from the critical point, the exponent attains lower values in systems with stronger screening and shorter range of the inter-particle interaction.

\section{Appendix A: Analytical values of $\alpha_{\mathrm{c}}$}

In a few simplest cases of high symmetry, when total energy of a cluster after a transition depends on a single generalized coordinate, values of a critical anisotropy parameter $\alpha_{\mathrm{c}}$ can be identified analytically. These are the transitions in 3-particle 2D system, 4-particle 3D cluster and all of the pyramidal transitions.

Consider the $N$-particle three-dimensional system undergoing a pyramidal $3 \mathrm{D} \rightarrow 2 \mathrm{D}$ dimensional transition. Immediately after the transition, a planar cluster consists of $n=N-1$ particles, positioned on the circumference of a circle with radius $R$, and a single particle in the center of confinement. The $2 \mathrm{D}$ cluster lies in $(x z)$ plane. The total potential energy of the system can be expressed as

$$
U_{0}(R)=\frac{1}{2} n R^{2}+\frac{n}{R} f(n)+\frac{n}{R} .
$$

A second term here represents the Coulomb interaction energy of $n$ particles positioned on the circle with radius $R$, thus forming a regular polygon. The function $f(n)$ depends only on the number of particles and is

$$
f(n)= \begin{cases}\frac{1}{4}+\frac{1}{2} \sum_{m=1}^{n / 2-1} \sin ^{-1}(m \pi / n) & \text { if } n \text { is even, } \\ \frac{1}{2} \sum_{m=1}^{(n-1) / 2} \sin ^{-1}(m \pi / n) & \text { if } n \text { is odd. }\end{cases}
$$

By setting $\partial U_{0} / \partial R=0$ we find the equilibrium radius to be simply

$$
R=(1+f(n))^{1 / 3}
$$

We further perturb the system by shifting the base by the small distance of $\delta y$, and, keeping the center of mass at the center of the confinement, an apex by the distance of $n \delta y$ in an opposite direction. Now, the total energy of the system is

$$
\begin{aligned}
U_{\mathrm{p}} & =\frac{1}{2} n\left(R^{2}+\alpha^{2} \delta y^{2}\right)+\frac{1}{2} \alpha^{2}(n \delta y)^{2} \\
& +\frac{n}{R} f(n)+\frac{n}{\sqrt{\left(R^{2}+(n+1)^{2} \delta y^{2}\right.}}
\end{aligned}
$$

By expanding the right hand side in powers of $\delta y$ up to the quadratic term, we get

$$
U_{\mathrm{p}} \approx U_{0}+\frac{1}{2} n \alpha^{2} \delta y^{2}(1+n)-\frac{n(n+1)^{2} \delta y^{2}}{2 R^{3}} .
$$

By requiring that

$$
\delta U=\frac{1}{2} n \alpha_{\mathrm{c}}^{2} \delta y^{2}(1+n)-\frac{n(n+1)^{2} \delta y^{2}}{2 R^{3}}=0
$$

for any small $\delta y$, and making use of equation A3, we find the critical value of anisotropy parameter to be

$$
\alpha_{\mathrm{c}}=\sqrt{\frac{N}{f(N-1)+1}} .
$$

Table I. Values of the function $f$ and critical parameter $\alpha_{\mathrm{c}}$ of $3 \mathrm{D}$ Coulomb systems undergoing dimensional transition of the pyramidal type.

\begin{tabular}{|c|c|c|}
\hline$N$ & $f(N-1)$ & $\alpha_{\mathrm{c}}$ \\
\hline \hline 5 & $\frac{1}{4}+\frac{\sqrt{2}}{2}$ & $2 \sqrt{\frac{5}{5+2 \sqrt{2}}} \approx 1.5983715$ \\
\hline 6 & $\sqrt{1+\frac{2}{\sqrt{5}}}$ & $\sqrt{3 \sqrt{5+2 \sqrt{5}}-\sqrt{5}} \approx 1.5889766$ \\
\hline 7 & $\frac{5}{4}+\frac{1}{\sqrt{3}}$ & $2 \sqrt{\frac{21}{27+4 \sqrt{3}}} \approx 1.5734727$ \\
\hline 8 & 2.3047649 & 1.5558750 \\
\hline
\end{tabular}

Values of the function $f(n)$ and corresponding critical parameters of pyramidal transitions are collected in table II for all 3D clusters with this type of structural transformation. We see, that in general, $\alpha_{\mathrm{c}}$ slightly decreases with $N$ in the range of $5-8$ particles.

In the two-dimensional case of $N=3$ particles, the system forms a triangular cluster. During the structural transition, one of its particles is pushed in-between the others, thus forming a linear structure. This case is basically a generalization of pyramidal transitions to the two-dimensions. Therefore, equation A7 is still valid and we find critical anisotropy to be $\alpha_{\mathrm{c}}=\sqrt{12 / 5}$, which is exactly the value observed in our numerical modelling.

A slightly different mechanism of transformation is observed in $3 \mathrm{D} \rightarrow 2 \mathrm{D}$ transition of highly symmetrical 
$N=4$ particle cluster. The final configuration is a $2 \mathrm{D}$ square, with potential energy

$$
U_{0}(R)=\frac{1}{2} N R^{2}+\frac{N}{R} f(N) .
$$

Minimization of A 8 by solving $\partial U_{0} / \partial R=0$ in turn gives $R=f(N)^{1 / 3}$. Right before the transition, two particles sharing a common diagonal in the final square are elevated by the distance of $\delta y$ above $(x z)$ plane, while other two are located at the same distance below it; ergo the energy of the perturbed system

$$
\begin{aligned}
U_{\mathrm{p}} & =\frac{1}{2} N\left(R^{2}+\alpha^{2} \delta y^{2}\right)+\frac{1}{4} \frac{N}{R}+\frac{N}{\sqrt{2 R^{2}+4 \delta y^{2}}} \approx \\
& \approx U_{0}+\frac{1}{2} N \alpha^{2} \delta y^{2}-\frac{N \sqrt{2} \delta y^{2}}{2 R^{3}} .
\end{aligned}
$$

By setting $\delta U=U_{\mathrm{p}}-U_{0}=0$, we again get

$$
\alpha_{\mathrm{c}}=\sqrt{\frac{\sqrt{2}}{f(4)}}=\sqrt{\frac{4 \sqrt{2}}{1+2 \sqrt{2}}} \approx 1.2155625 .
$$

This value is exactly the same as found by our numerical procedure.
[1] A. J. Koch and H. Meinhardt, Rev. Mod. Phys. 66, 1481 (1994).

[2] S. Wolfram, Rev. Mod. Phys. 55, 601 (1983).

[3] A. J. Chakravarti, G. Baumgartner, and M. Lauria, IEEE Trans. Syst., Man, Cybern. A, Syst., Humans 35, 373 (2005).

[4] H. Ikegami, H. Akimoto and K. Kono, Phys. Rev. Lett. 102, 046807 (2009)

[5] C. C. Grimes and G. Adams, Phys. Rev. Lett. 42, 795 (1979).

[6] I. Waki, S. Kassner, G. Birkl, and H. Walther, Phys. Rev. Lett. 68, 2007 (1992).

[7] M. Bonitz, C. Henning and D. Block, Rep. Prog. Phys. 73, 066501 (2010)

[8] A. Schella, T. Miksch, A. Melzer, J. Schablinski, D. Block, A. Piel, H. Thomsen, P. Ludwig, and M. Bonitz, Phys. Rev. E 84, 056402 (2011).
[9] V. Nosenko, S. K. Zhdanov, A. V. Ivlev, C. A. Knapek, and G. E. Morfill, Phys. Rev. Lett. 103, 015001 (2009).

[10] A. Melzer, Phys. Rev. E 73, 056404 (2006).

[11] T. E. Sheridan and K. D. Wells, Phys. Rev. E 81, 016404 (2010).

[12] A. Radzvilavičius and E. Anisimovas, J. Phys.: Condens. Matter 23, 075302 (2011).

[13] N. Metropolis, A. W. Rosenbluth, M. N. Rosenbluth, A. H. Teller, and E. Teller, J. Chem. Phys. 21, 1087 (1953).

[14] H. Kahlert, P. Ludwig, H. Baumgartner, M. Bonitz, D. Block, S. Kading, A. Melzer, and A. Piel, Phys. Rev. E 78, 036408 (2008).

[15] M. Kong, B. Partoens, and F. M. Peeters, Phys. Rev. E 65, 046602 (2002).

[16] M. Saint Jean, C. Even and C. Guthmann, Europhys. Lett. 55, 45 (2001).

[17] O. Rancova, E. Anisimovas and T. Varanavičius, Phys. Rev. E 83, 036409 (2011). 